\title{
Strain Measurement of Moving Periodic Objects With Optical Sensors
}

\author{
Christian Berger
}

\begin{abstract}
Measuring the strain of an object provides information about axial loads which it is exposed to. In some applications, this information is wanted not from still-standing, but from linearly moving objects. Measuring the strain of V-belts, e.g., permits investigating the operational behavior of combustion engine belt drives. For this purpose, new noncontact measurement methods using optical sensors are being developed. The sensors detect periodic patterns on the moving object. The strain is determined from a frequency analysis of the sensor signal.
\end{abstract}

Index Terms-Periodic patterns, strain measurement, V-belt, velocity measurement.

\section{INTRODUCTION}

A PERIODIC structure on the surface of an expandable object can provide information about the strain of the object [1]. When the strain $\varepsilon$ varies, the cycle length $c_{x}$ of the structure changes accordingly

$$
c_{x}=c_{x, r} \cdot(1+\varepsilon)
$$

( $c_{x, r}$ : reference cycle length). The cycle lengths may be evaluated, e.g., by image processing methods. Objects with nonperiodic surfaces have to be marked with artificially coded patterns.

When the object observed is moving, adequate exposure times may be used [2] in order to avoid unsharpness of the images to be processed. However, observation of small $\varepsilon$-variations at high object speeds would require very short exposure times. In the particular case of linear object motion, this problem can be avoided with a measurement method presented in this contribution. It is based on using optical sensors capable of resolving the periodic pattern of the object. The time-dependent sensor signal is thus periodic, too, and information about strain variations can be obtained from evaluating the signal frequency.

The paper is organized as follows: Section II describes how strain variations of moving periodic objects can be concluded from the frequency of a sensor signal. The demands on the design of optical sensors are outlined in Section III. Section IV gives an overview of noncontact velocity measurement, which is needed to scale the measured temporal frequency down to the spatial frequency indicating the belt strain. Section V proposes different methods of strain measurement. One of them, which is based on the combination of frequency evaluation and correlative velocimetry, is experimentally verified in Section VI.

Manuscript received May 29, 2001; revised April 22, 2002.

The author is with the Institut für Mess-und Regelungstechnik, Universität Karlsruhe, Karlsruhe, Germany (e-mail: Christian.Berger@mrt.uka.de).

Digital Object Identifier 10.1109/TIM.2002.802252

\section{StRain MEASUREMENT OF LinEARLy MOVING OBJECTS}

In the following, an almost periodic surface pattern consisting of straight lines is assumed [see Fig. 1(a)]. The lines are inclined at an angle $\varphi$ relative to the $x$-axis, which is the direction of object motion. The pattern can thus be described by an intensity distribution $i(\mathbf{x})(\mathbf{x}=(x, y)$ : spatial coordinates), which satisfies the following equation:

$$
\begin{aligned}
i(\mathbf{x}-k \cdot \mathbf{c}) & =i\left(\mathbf{x}-k \cdot \frac{\mathbf{c}}{|\mathbf{c}|} \cdot c_{x} \sin \varphi\right) \\
& \approx i(\mathbf{x}), \quad k=0, \pm 1, \pm 2, \ldots
\end{aligned}
$$

As outlined in Fig. 1(a), c denotes the vector of the cycle length of the pattern, and $c_{x}$ is the cycle length in $x$-direction. The corresponding Fourier spectrum $I(\mathbf{f})=\mathcal{F}^{2}\{i(\mathbf{x})\}$ (f $=\left(f_{x}, f_{y}\right)$ : spatial frequencies, $\mathcal{F}^{2}\{\cdot\}$ : two-dimensional Fourier transform) has a maximum peak at the positive frequency

$$
\mathbf{f}_{\mathbf{c}}=\left(f_{x, c}, f_{y, c}\right)=\frac{1}{|\mathbf{c}|} \cdot \frac{\mathbf{c}}{|\mathbf{c}|}=\frac{1}{c_{x}} \cdot(1,-\cot \varphi)
$$

and upper harmonics at multiples of $\mathbf{f}_{\mathbf{c}}$, as outlined in Fig. 1(b).

When the object is moving at a velocity $\mathbf{v}=(v, 0)$, an ideal sensor, capable of resolving all pattern details, would generate a time-dependent periodic signal $s(t)$ with a main frequency

$$
f_{c}=\mathbf{v} \cdot \mathbf{f}_{\mathbf{c}}=v \cdot f_{x, c}=\frac{v}{c_{x}}
$$

in its Fourier spectrum $S(f)$ [see Fig. 1(c)].

Equation (4) shows that the cycle length of the pattern and the main frequency of the resulting signal are related by the object velocity. Thus, if $v$ and $f_{c}$ are both measured, the strain of the object can be concluded from (1) and (4)

$$
\varepsilon=\frac{\frac{v}{f_{c}}}{c_{x, r}}-1
$$

where $c_{x, r}$ has to be determined by a reference measurement.

Besides the need of independent measurements of $f_{c}$ and $v$, the following conditions have to be satisfied in order to avoid a detection of $\mathbf{f}_{\mathbf{c}}$-variations which do not result from strain changes.

- The direction of the velocity vector $\mathbf{v}$ must be constant.

- In case of uniform strain distribution along the object, the cycle vector $\mathbf{c}$ of the periodic pattern must be constant.

- The angle $\varphi$ must not be close to or equal $0^{\circ}$. 

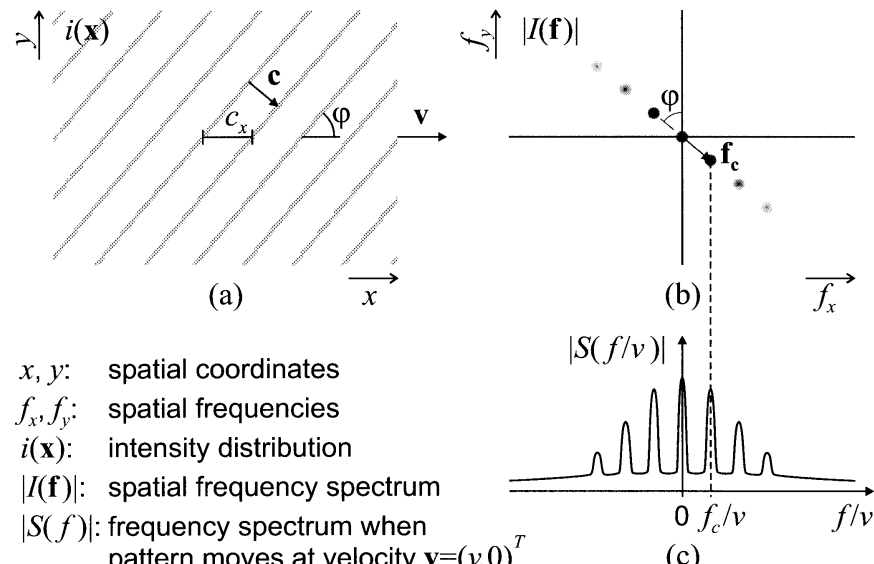

$x, y: \quad$ spatial coordinates

$f_{x}, f_{y}:$ spatial frequencies

$i(\mathbf{x})$ : intensity distribution

$|I(\mathbf{f})|$ : spatial frequency spectrum

$|S(f)|$ : frequency spectrum when

pattern moves at velocity $\mathbf{v}=(v, 0)^{T}$

(c)

Fig. 1. Signal generation from moving periodic patterns.

\section{Detection of Periodic Structures on V-Belts BY OPTICAL SENSORS}

For the special application of strain measurement of combustion engine V-belts, the preconditions mentioned above are met:

- The V-belt is guided by pulleys in the belt drive. Hence, the direction of the belt velocity is constant.

- Usually, V-belts have a periodic structure consisting of crossed lines on their back surface (see Fig. 2). The lines are equally spaced with a cycle length of $c_{x} \approx 0.9 \mathrm{~mm}$ on common V-belts. When this structure is adequately illuminated by directional lighting, only one of the line groups appears at high contrast [cf. Fig. 2(b)], resulting in a pattern, as shown in Fig. 1(a).

Thus, no artificially coded patterns are needed for strain measurement of common V-belts. Instead, the structure of the belt itself can be evaluated by optical sensors.

In general, an optical sensor consists of a detector with aperture function $a(\mathbf{x})$, and of an optical imaging system with magnification $M$. For $\mathbf{v}=(v, 0)$, the frequency spectrum of the sensor signal is given by the following equation [3], [4]

$$
\begin{aligned}
|S(f)|=\frac{C}{M^{2} v} \cdot \int_{-\infty}^{\infty}\left|O\left(\frac{f}{M v}, \frac{f_{y}}{M}\right)\right| \\
\cdot\left|A\left(\frac{f}{M v}, \frac{f_{y}}{M}\right)\right| \cdot\left|I\left(\frac{f}{v}, f_{y}\right)\right| d f_{y}
\end{aligned}
$$

( $C$ is constant). Equation (6) shows that the sensor signal is obtained from the pattern spectrum $I(\mathbf{f})$, filtered with the spatial frequency response $A(\mathbf{f})=\mathcal{F}^{2}\{a(\mathbf{x})\}$ of the detector and the optical transfer function $O(\mathbf{f})$ [5] of the lens system. Assuming perfectly focused optical imaging and neglecting diffraction effects, $O(\mathbf{f} / M)$ is unity. In contrast, $A(\mathbf{f} / M)$ acts as a spatial low-pass filter on the pattern spectrum, which can result in an unwanted shift of the measured signal frequency [6]. In order to avoid this peak shift, $M \cdot\left|\mathbf{f}_{\mathbf{l}}\right| \gg\left|\mathbf{f}_{\mathbf{c}}\right|$ should be satisfied, where $\mathbf{f}_{\mathbf{1}}$ denotes the limiting frequency of $A$.

Moreover, (6) shows that the signal spectrum at a given frequency $f$ is obtained from an integration of all filtered $I$-values with arguments $\mathbf{v} \cdot \mathbf{f}=f$. Thus, each point of the signal

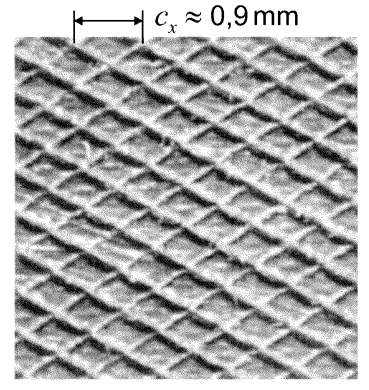

(a)

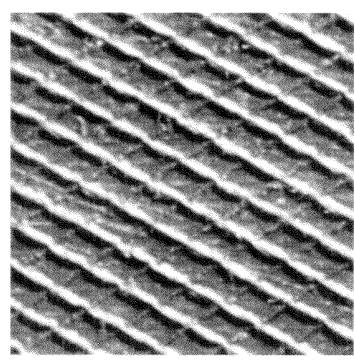

(b)
Fig. 2. Macro images of V-belt back surface obtained with (a) diffuse illumination and with (b) directional illumination.

spectrum $S$ corresponds to a line of $I$-values perpendicular to the direction of $\mathbf{v}$ [cf. Fig. 1(b) and (c)]. Hence, in order to avoid an unwanted widening of the spectral peak at $f=f_{c}$, $I(\mathbf{f})$ should not contain dominant components-except the main peak itself-with arguments $f_{x} \approx f_{x, c}$. On V-belts, such components are caused by a second line group inclined at an angle $\varphi^{\prime} \approx-\varphi$ [see Fig. 2(a)]. Therefore, it is advantageous to reduce the contrast of one of the line groups by illuminating the V-belt directionally [see Fig. 2(b)].

Concerning the shape of $a(\mathbf{x})$, it has to be considered that only circular functions have isotropic two-dimensional Fourier transforms. Therefore, detectors with circular aperture functions are favorable if the angle $\varphi$ of the pattern lines may vary on different types of V-belts.

\section{Velocity Measurement}

Besides the prerequisite of frequency evaluation of the sensor signal, strain measurement with the method proposed in Section II requires that the object velocity is measured. For a reliable detection of small strain variations, high accuracy of the measured values is needed. In particular, the measured value of the velocity must not be affected by slippage, which is frequently occurring in belt drives [7]. Non-contact velocimetry offers the desired properties. Correlation or spatial filter methods are well-known sensing principles, which will be described in the following.

\section{A. Velocity Measurement With Correlation Sensors}

For velocity measurement by correlation techniques [8], two identical detectors are aligned at a known distance $L$ in the direction of object motion (see Fig. 3). In the ideal case, the signals of these detectors are identical, except for a shift by the transit time $T$

$$
s_{2}(t)=s_{1}(t-T)
$$

From this, the velocity

$$
v=\frac{L}{T} .
$$

can be concluded. To measure $T$, the cross-correlation function [9]

$$
\Phi_{12}(\tau)=E\left\{s_{1}(t-\tau) \cdot s_{2}(t)\right\}
$$


$(E\{\cdot\}:$ expected value) of the sensor signals is evaluated. Since this function has a maximum at $\tau=T$ for signals satisfying (7), $T$ can be obtained from tracking the maximum position of $\Phi_{12}(\tau)$. This is accomplished, e.g., by a digital correlator described in [10].

It is evident that the maximum position is not changing when the signals $s_{1}$ and $s_{2}$ are periodic. However, if the cross-correlation function is periodic, care has to be taken that the correlator is not tracking a side maximum of $\Phi_{12}$. This can be avoided by providing supplementary information about the approximate object speed, which is available, e.g., from rotational speeds of pulleys.

\section{B. Velocity Measurement With Spatial Filter Sensors}

Velocity measurement by spatial filtering requires an optical setup with a grating structure in the direction of object motion [11]. The grating is realized, e.g., by a linear array of detectors. Its aperture function $a_{0}(\mathbf{x})$ and spatial frequency response $A_{0}(\mathbf{f})=\mathcal{F}^{2}\left\{a_{0}(\mathbf{x})\right\}$ are shown in Fig. 4. Obviously, the grating acts as a spatial bandpass filter. The main passband of $A_{0}$ is located at the spatial frequency $f_{x, 0}=1 / g_{0}$, where $g_{0}$ denotes the period of the detector array. Furthermore, the pass-band is characterized by the peak width $\Delta f_{x}$ in direction of the grating, and by the width $\Delta f_{y}$ in perpendicular direction. They are depending on the number $N$ of grating periods and the element height $h$, respectively

$$
\frac{\Delta f_{x}}{f_{x, 0}} \propto \frac{1}{N}, \quad \Delta f_{y} \propto \frac{1}{h} .
$$

In the example of Fig. $4, N=10$ and $h=g_{0} / 2$.

Assuming perfectly focused optical imaging with magnification $M_{0}$, the frequency spectrum of the spatial filter signal $s_{0}$ is obtained from [cf. (6)]

$$
\begin{aligned}
\left|S_{0}(f)\right|=\frac{C}{M_{0}^{2} v} \cdot \int_{-\infty}^{\infty}\left|A_{0}\left(\frac{f}{M_{0} v}, \frac{f_{y}}{M_{0}}\right)\right| \\
\cdot\left|I\left(\frac{f}{v}, f_{y}\right)\right| d f_{y}
\end{aligned}
$$

where $C$ is a constant. The main temporal frequency $f_{0}$ of the signal is related to the object velocity, the optical magnification and the passband frequency $\mathbf{f}_{0}=\left(f_{x, 0}, f_{y, 0}\right)$ of $A_{0}$ according to

$$
f_{0}=M_{0} \cdot \mathbf{v} \cdot \mathbf{f}_{\mathbf{0}}=M_{0} \cdot v \cdot f_{x, 0}=M_{0} \cdot \frac{v}{g_{0}} .
$$

This equation shows that $v$ can be obtained from measuring $f_{0}$, provided that $M_{0}$ and $g_{0}$ are given.

Whereas the frequency $f_{c}$ discussed in Section III resulted from periodic patterns on the object to be measured, the frequency $f_{0}$ is generated by the periodic detector structure. Therefore, velocity measurement with spatial filter sensors does not require periodic object patterns, but is practicable whenever the spectrum $I(\mathbf{f})$ contains spatial frequency components in the passband of $A_{0}\left(\mathbf{f} / M_{0}\right)$.
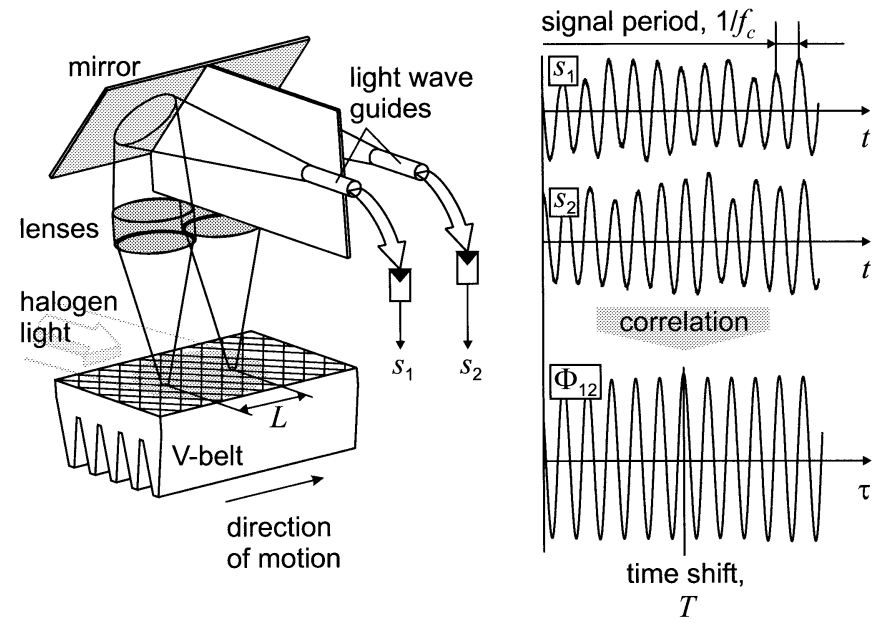

Fig. 3. Compact optical sensor for strain measurement of V-belts.
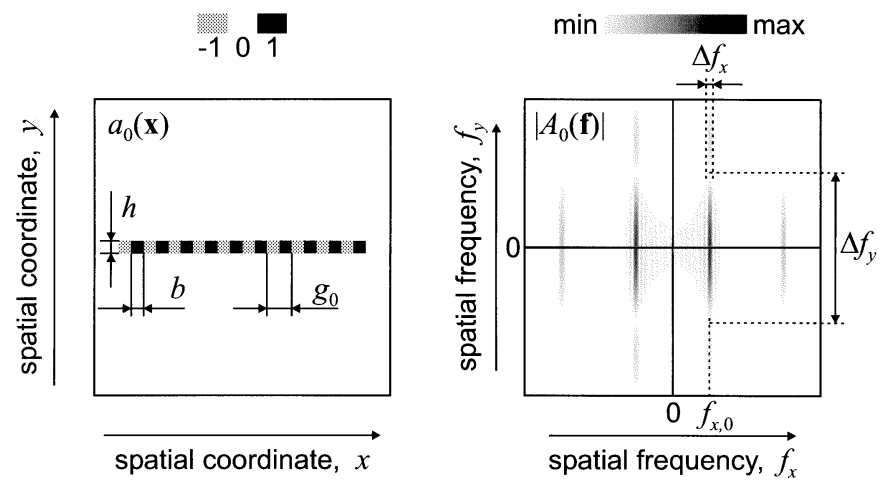

Fig. 4. Aperture function and spatial frequency response of a spatial filter sensor.

\section{Design of Strain Sensors Combining Frequency AND VELOCITY MEASUREMENT}

In order to obtain information about the strain of a moving object, frequency and velocity measurements have to be combined. For practical reasons, both measured values $f_{c}$ and $v$ should be obtained from one single sensor. In the following, the design of optical sensors using velocity measurement by either correlation or spatial filter methods will be discussed.

\section{A. Strain Measurement With Correlation Sensors}

As outlined in Section III, a single optical detector can transform the main spatial frequency of a moving periodic pattern into a periodic time-dependent signal, provided that the main peak of the frequency spectrum $I(\mathbf{f})$ is within the passband of the detector's frequency response $A(\mathbf{f} / M)$. In Section IV-A, it was stated that velocity measurement by correlation techniques is possible even if two time-shifted signals are perfectly periodic, provided that additional information about the approximate velocity is available.

Hence, a compact strain sensor can be built by aligning two identical optical detectors, each of which is capable of resolving the periodic object structure. The optical front-end of a prototype designed for measurements on V-belts [7], [12] is shown in Fig. 3. The V-belt is illuminated by directional light emitted 
from a halogen lamp. The reflected radiation is passing the optical system and is then transmitted by polymer optical fibers to the electrical signal processing unit. The digital correlator is provided with supplementary information about the V-belt velocity, obtained from rotary encoders at pulleys in the belt drive.

From the measured values of the time shift $T$ and the signal main frequency $f_{c}$, strain variations can be calculated by evaluating

$$
\varepsilon=\frac{L}{T \cdot f_{c} \cdot c_{x, r}}-1=\frac{T_{r}}{T} \cdot \frac{f_{c, r}}{f_{c}}-1
$$

where $T_{r}$ and $f_{c, r}$ denote reference values measured at the initial belt strain.

An advantage of the measurement method proposed is that strain information is obtained from a simple optical sensor setup. However, the fact that two quantities measured independently from each other have to be combined is disadvantageous, because the individual measuring errors of $T$ and $f_{c}$ add up and may hence distort the result of (13) severely. This effect can be observed in the results of measurement series presented in Section VI.

\section{B. Strain Measurement With Spatial Filter Sensors}

In the following, methods for strain measurement with spatial filter sensors will be described. Like in the Sections III and IV-B, the aperture functions of a single detector and of a detector array will be denoted by $a(\mathbf{x})$ and $a_{0}(\mathbf{x})$, respectively. One common optical system with magnification $M_{0}$ for both the single detector and the detector array shall be assumed.

Whereas the spatial frequency $f_{x, c}$ is determined by the cycle length of a periodic pattern [cf. (3)], the passband frequency $f_{x, 0}$ of a spatial filter sensor can be varied by design of the optical parameters $g_{0}$ and $M_{0}$ [cf. (12)]. Therefore, design of the latter parameters decides on whether the two frequencies coincide or not. Variations of the object strain can be detected with both configurations, as will be shown in the following.

1) Coincidence of the Spatial Frequencies of the Surface and the Filter Passband: A maximum amplitude of the sensor signal is obtained if the main frequency peak of $I(\mathbf{f})$ is located within the passband of $A_{0}\left(\mathbf{f} / M_{0}\right)$, as outlined one-dimensionally in Fig. 5(a). Hence, the spatial frequencies $M_{0} f_{x, 0}$ and $f_{x, c}$ have to coincide

$$
M_{0} \cdot f_{x, 0}=\frac{M_{0}}{g_{0}} \stackrel{!}{=} f_{x, c} .
$$

In order to satisfy this requirement, the detector parameter $M_{0} / g_{0}$ has to be varied proportional to $f_{x, c}$, which in turn is depending on the strain $\varepsilon$. Hence, the amplitude of the sensor signal can be maximized, e.g., by adapting the sensor's optical magnification $M_{0}$. Then, $\varepsilon$ is obtained from [cf. (4), (5), and (12)]

$$
\varepsilon=\frac{g_{0}}{M_{0} c_{x, r}}-1=\frac{M_{0, r}}{M_{0}}-1,
$$

where $M_{0, r}$ denotes the reference magnification set at the initial belt strain.
Particular care has to be taken if the peak coordinate $f_{y, c}$ of $I$ is nonzero, i.e., if the pattern consists of lines with $\varphi \neq 90^{\circ}$ (cf. Fig. 1). In this case, an adequately small detector height $h$ has to ensure that the passband of $A_{0}(\mathbf{f} / M)$ is sufficiently broad in $y$-direction [cf. (10)]. Since narrow detectors imply a high sensitivity of the sensor to angular misalignment [3], the grating must be accurately aligned in the direction of motion, in order to avoid an unwanted widening of the spectral peak of the signal.

The application of the measurement method proposed requires that the signal amplitude is significantly changing even if the strain is only slightly varying. This requirement is only satisfied for large $N$-values of the grating, leading to a passband with narrow bandwidth $M_{0} \Delta f_{x}$ [cf. (10)]. Furthermore, an ambiguity of the method proposed has to be considered: Assuming a spatial filter adjusted to satisfy (14) at an initial strain, deviations of the strain from the initial value are indicated by decreasing signal amplitude, independent from whether the strain decreases or increases. In order to avoid this ambiguity, the sensor adjustment has to be modified. Instead of satisfying (14), the parameter $M_{0} / g_{0}$ can be set, e.g., to satisfy

$$
f_{x, 0}=\frac{M_{0}}{g_{0}} \stackrel{!}{=} f_{x, c}+\frac{M_{0} \Delta f_{x}}{4} .
$$

As outlined in Fig. 5(b), the surface frequency $f_{x, c}$ then coincides not with the maximum, but with the rising edge of the detector passband, thus leading to some intermediate signal amplitude. Hence, changes of the strain are indicated unambiguously by amplitude variations that are approximately proportional to the variations of $f_{x, c}$.

It is obvious that the measurement method just described can also be realized with a spatial low- or high-pass filter [cf. Fig. 5(b)]. Equivalent to the considerations just given, the cut-off frequency of the filter has to be adjusted to coincide with $f_{x, c}$, and the filter frequency response should have maximum edge steepness at the cut-off frequency.

2) Different Spatial Frequencies of the Surface and the Filter Passband: If $I(\mathbf{f})$ has a main frequency peak outside, but other nonzero components inside the passband of $A_{0}\left(\mathbf{f} / M_{0}\right)$, two signal frequencies $f_{0}$ and $f_{c}$ can be generated. They result from the periodic structure of the spatial filter and from the periodic pattern, respectively. To detect the frequency $f_{c}$, the signal from either one of the grating detectors or from a separate, adequately shaped detector may be evaluated. Information about the strain is obtained from comparing the two frequencies

$$
\varepsilon=\frac{g_{0}}{M_{0} c_{x, r}} \cdot \frac{f_{0}}{f_{c}}-1=\frac{f_{0}}{f_{0, r}} \cdot \frac{f_{c, r}}{f_{c}}-1 .
$$

Equivalent to (13) and (15), $f_{c, r}$ and $f_{0, r}$ denote reference frequencies measured at the initial belt strain.

With the measurement method proposed, information about variations of the object strain is obtained from frequency measurement. When applying this method, the frequency $f_{0}$ generated by the spatial filter sensor must not be affected by variations of $\varepsilon$. Hence, the sensor parameters $M_{0}$ and $g_{0}$ have to be adequately designed in order to ensure that $M_{0} f_{x, 0}$ does not coincide either with the main frequency $f_{x, c}$ of the pattern, or with upper harmonics of $f_{x, c}$. If the pattern consists of inclined lines, its influence on the spatial filter signal can be 
(a)

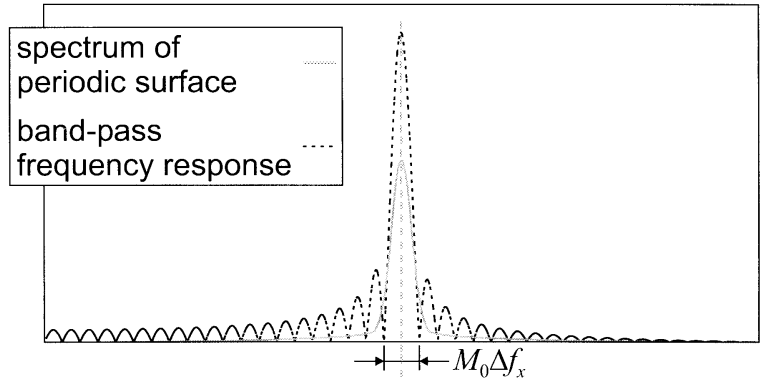

(b)

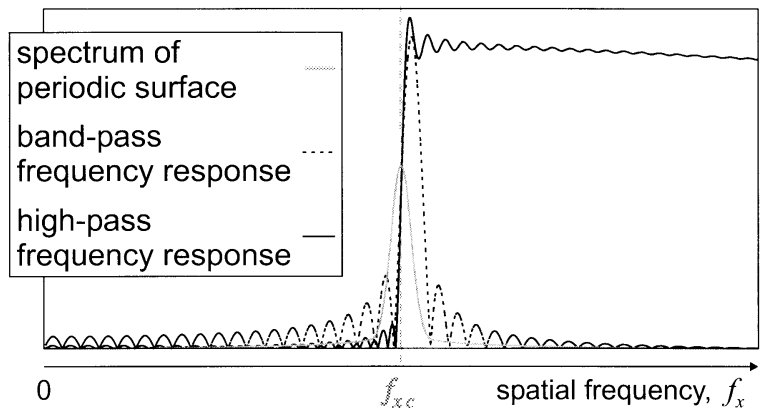

Fig. 5. Spatial frequency peak of a periodic pattern coinciding with maximum (a) and with rising edge (b) of frequency responses of spatial filters.

further reduced by designing a grating with broad detector dimensions $h$, leading to a narrow bandwidth $M_{0} \Delta f_{y}$ of the spatial filter frequency response [cf. (10)]. This also reduces the sensitivity to angular misalignment of the spatial filter sensor.

\section{Results of StRain Measurement of V-Belts}

To determine the strain of moving V-belts, several measurement series have been carried out with the correlation system described in Section V-A. Defined values of the belt strain were set by varying the position of one pulley at simple V-belt test rigs.

A first measurement series was carried out at a test rig where the axial load of the belt was measured. The V-belt circulated at a speed of $9.1 \mathrm{~m} / \mathrm{s}$ during the test. The measured average strain values are almost linearly dependent on the belt force [see Fig. 6]. However, the span of the single measurements, illustrated by the vertical bars in Fig. 6, is large. As explained in Section V-A, the severe deviations from the averaged strain values result from the individual measuring errors of the frequency and the velocity, which add up in the result of (13). Hence, the measurement method described in Section V-A is not suited for real-time strain measurement, but requires a considerable averaging of the measured values.

A second measurement series was carried out at a V-belt speed of $2 \mathrm{~m} / \mathrm{s}$ on a simpler test rig, where no information about the belt load was available. In order to prove the reliability of the measured strain values, they were compared to the set values, which were obtained from measuring the pulley displacement. The result shows a quite exact correspondence between the measured average strain values and the set values (see Fig. 7).

The results of the measurement series prove that the periodic structure on common V-belts can be used for the detection of changes of the belt strain. Due to the large elastic modulus of approx. $3000 \mathrm{~N} / \mathrm{mm}^{2}$, which was measured in a tension test of

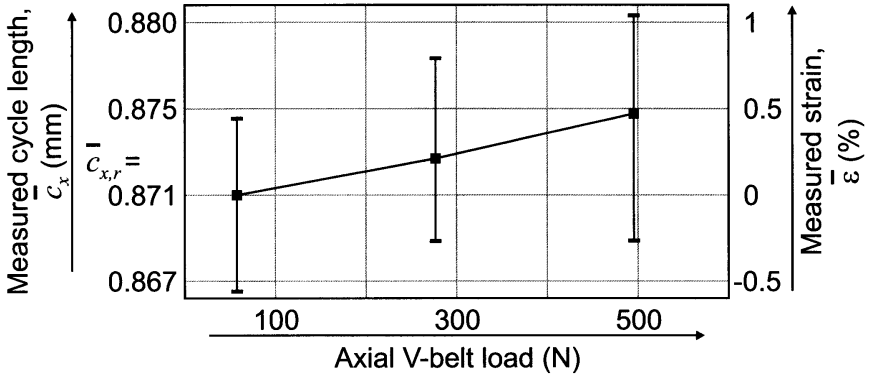

Fig. 6. Strain measurement of V-belt (setting of axial belt load)

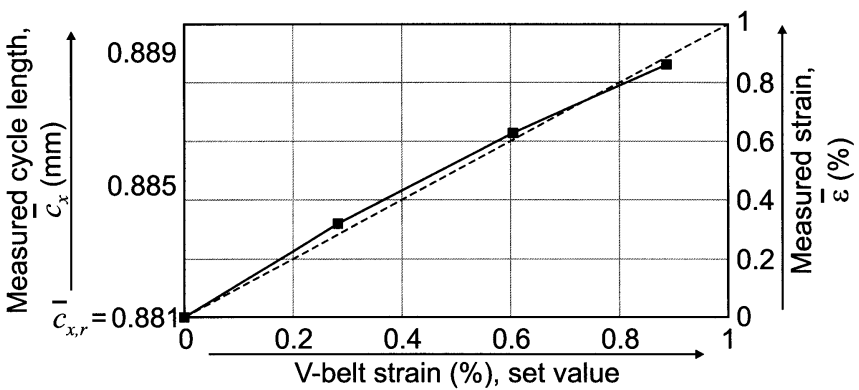

Fig. 7. Strain measurement of V-belt (setting of defined belt strain).

a common V-belt [12], only small strain of at most one or two percent occurs even at high axial V-belt load. Hence, the resolution of the strain values must be in an order of magnitude of $0.1 \%$ or lower, thus requiring a high accuracy of the measured frequency and velocity values.

A detection of considerable strain changes of moving V-belts may indicate excessive axial loads, pointing to an unfavorable operating behavior of the belt drive, or slackness of the belt due to aging effects.

\section{SUMMARY}

In this paper, methods for contactless measurement of the strain of moving objects have been proposed. The measurement methods require that the object has a periodic pattern which can be detected by optical sensors. Then, the frequency of the signal generated by a single detector is dependent on the strain of the object. As the frequency is also scaled with the object speed, velocity information is needed to scale the measured temporal frequencies down to spatial frequencies, from which the strain can be concluded. For this purpose, noncontact velocimetry using correlation or spatial filter techniques may be applied. In case of spatial filter sensors, design of the optical parameters decides on whether the spatial frequency of the surface is within the detector passband or not, resulting in different strategies for the detection of strain variations.

The measurement method based on a combination of correlative velocimetry and frequency evaluation has been experimentally verified with $\mathrm{V}$-belts. The results of various measurement series show that the periodic structure of V-belts can be used to obtain information about changes of the belt strain. As the strain variations occurring at $\mathrm{V}$-belts are small, however, the statistical variations of the measured values of frequency and velocity lead to large statistical errors of the measured strain. The measurement method described in Section V-B-I may lead to a reduc- 
tion of these errors, because it is based on the evaluation of one spatial filter signal, instead of combining two values measured independently from each other.

\section{REFERENCES}

[1] A. J. Durelli and V. J. Parks, Moiré Analysis of Strain. Englewood Cliffs, NJ: Prentice-Hall, 1970.

[2] W. Vocke and K. Ullmann, Experimentelle Dehnungsanalyse-Dehngitterund Moiréverfahren. Leipzig, Germany: VEB Fachbuchverlag, 1974.

[3] A. Hayashi and Y. Kitagawa, "Image velocitiy sensing using an optical fiber array," Appl. Opt., vol. 21, no. 8, pp. 1394-1399, 1982.

[4] R. Fritsche, "Vergleich berührungsloser Geschwindigkeitsmessverfahren an selbstleuchtendem und inkohärent beleuchtetem Walzgut," Ph.D., Univ. Karlsruhe, Karlsruhe, Germany, 1979.

[5] J. W. Goodman, Introduction to Fourier Optics. New York: McGrawHill, 1968.

[6] Ch. Berger, "Optical sensors for strain measurement on moving V-belts," in Proc. 18th IEEE Instrum. Meas. Technol. Conf., Budapest, Hungary, 2001, pp. 266-271.

[7] Ch. Berger, K.-H. Hanus, A. Wehle, and O. Wüstefeld, "Bestimmung des scheibenspezifischen Schlupfes in Kraftfahrzeug-Riementrieben," MTZ Motortechnische Zeitschrift, vol. 63, no. 1, pp. 46-51, 2002.
[8] F. Mesch, "Speed and flow measurement by an intelligent correlation system," in Proc. ISA, New Orleans, LA, 1990, pp. 1899-1914.

[9] A. Papoulis, Probability, Random Variables, and Stochastic Processes, Singapore: McGraw-Hill, 1984

[10] R. Zeitler, "Digital correlator for measuring the velocity of solid surfaces," IEEE Trans. Instrum. Meas., vol. 46, pp. 803-806, Aug. 1997.

[11] O. Fiedler, J. Werther, N. Labahn, J. Kumpart, and K. Christofori, "Measurement of local particle velocities and velocity distributions in gassolid flows by means of the spatial filter method," Powder Technol., vol. 94, no. 1, pp. 51-57, 1997.

[12] A. Wehle, "Berührungslose Messung dynamischer Belastungskenngrößen in Kfz-Riementrieben,” M.S., Univ. Karlsruhe, Karlsruhe, Germany, 2000

Christian Berger received the M.S. degree in mechanical engineering from the University of Karlsruhe, Karlsruhe, Germany, in 1997.

Since 1998, he has been with the Institut für Mess- und Regelungstechnik as a Research Assistant. His research interests include optical sensors for noncontact velocimetry and correlation methods. His current work focuses on optimization of the optical and electronic design of such sensors to avoid systematic measuring errors. 